Polymer Journal, Vol. 7, No. 1, pp 8-13 (1975)

\title{
Correlation between ESCA and NMR Chemical Shifts of Carbons in Linear Polymers
}

\author{
Shoji Suzuki, Yoshio Inoue, Isao Ando, Riichirô ChûJô \\ Department of Polymer Chemistry, Tokyo Institute of Technology, \\ Ookayama, Meguro-ku, Tokyo 152, Japan. \\ Kazuyoshi Seto, and Kiyoshi Hoshino \\ Kokusai Electric Co., Ltd., 1-1 Shimmeidai 2-chome, Hamura-cho, \\ Tokyo 190-11, Japan.
}

(Received February 6, 1974)

\begin{abstract}
ESCA spectra in CK region were measured for several kinds of linear polymers; corrections for the influence of charging were made. The chemical shifts $\left(\Delta E_{\mathrm{b}}\right)$ in the ESCA spectra observed were compared with the chemical shifts $\left(\delta_{\mathrm{c}}\right)$ in ${ }^{13} \mathrm{C}$ NMR and electron densities $(E D)$. There are generally good linearities in both comparisons with only a few exceptions. Two kinds of linear relations can be deduced in the ESCA data and one in the ED. One ESCA relation is deduced from all the data observed and the other from the data of the aliphatic groups alone. The linear relations are:
\end{abstract}

$$
\begin{array}{ll}
\Delta E_{\mathrm{b}}=-0.029 \delta_{\mathrm{c}}+4.35 & \text { (whole) } \\
\Delta E_{\mathrm{b}}=-0.045 \delta_{\mathrm{c}}+6.85 & \text { (aliphatic) } \\
\Delta E_{\mathrm{b}}=-9.43 E D+37.38 &
\end{array}
$$

We can safely say that there are linear relations between CK ESCA and ${ }^{13}$ C NMR chemical shifts, and between ESCA chemical shifts and electron densities. These linear relations serve for the assignment of peaks in the course of ESCA studies.

KEY WORDS Electron Spectra / ESCA / NMR / Electron Density / Aliphatic Group / Poly(vinyl alcohol) / Polystyrene / Contamination / Charging / Evaporation Method /

ESCA (Electron Spectroscopy for Chemical Analysis) is one of the analytical instruments which has rapidly advanced in recent years. It is a technique for determining the binding energies of inner shell electrons so that the chemical structure of a molecule can be determined from its chemical shift. ESCA seems to be a very useful technique for obtaining information on polymer configuration and conformation when it is applied to polymer systems. In order to analyse ESCA spectra precisely the assignment of each peak in the spectra to a corresponding configuration or conformation must be carried out. Such assignments have not been completed as yet. One of the purposes of this report is to obtain some information about assignments of ESCA chemical shifts by using
NMR (Nuclear Magnetic Resonance) chemical shifts. Several investigations have been made to study the correlation between ESCA and NMR chemical shifts in low-molecular-weight compounds. $^{1-4}$ We will also examine the correlation between ESCA chemical shifts and electron densities, because it can be expected that the binding energy will decrease when the electron density around the atom increases. There have been only a few ESCA studies of polymers; polyethylene and others, ${ }^{5}$ the helixcoil transition of poly( $\gamma$-benzyl L-glutamate $),{ }^{6}$ the crystallinity of fluorine-containing polymers, ${ }^{7}$ the surface oxidation of wool ${ }^{8}$, fluorinecontaining polymers, ${ }^{9,10}$ and nitroso rubbers. ${ }^{11}$

The fact that there have been only a few studies about the ESCA spectroscopy of polymers 
implies that the comparison of ESCA chemical shifts with NMR chemical shifts and electron densities can assist in the assignment of peaks observed in the course of ESCA research.

\section{EXPERIMENTAL}

\section{Apparatus}

ESCA spectra were measured with a Kokusai/ AEI ES-100 electron spectrometer.

\section{Sample}

The sample polymers were the ones used in the ${ }^{13} \mathrm{C}$ NMR study, for convenience in comparison with NMR chemical shifts. Detailed descriptions are given elsewhere ${ }^{12-18}$ except for poly(ethylene glycol). Poly(ethylene glycol), lot 805130, was purchased from Nippon Oil \& Fats Co., Ltd. Samlpes used are as follows:

Polyacrylonitrile (PAN), poly(vinyl alcohol) (PVA), poly(methyl methacrylate) (PMMA), poly (vinyl chloride) (PVC), polystyrene (PSt), poly (ethylene glycol) (PEG), polyethylene (PE), poly (vinyl acetate) (PVAc) and polypropylene (PP). All samples were used in solid state, whose forms are as follows:
powder......PAN, PVC, PSt, PEG, PP

bulk ........PVA, PMMA, PVAc

grain.......PE

\section{Procedure}

The aluminum $\mathrm{K}-\alpha_{1,2}(1486.6 \mathrm{eV})$ and the magnesium $\mathrm{K}-\alpha_{1,2}(1253.6 \mathrm{eV})$ radiation were used as the X-ray excitation sources. The X-ray power supply was run at $12 \mathrm{kV}$ and $25 \mathrm{~mA}$. Spectra were measured at room temperature.

\section{RESULTS AND DISCUSSION}

Typical CK ESCA spectra are reproduced in Figures 1-3. The CK ESCA chemical shifts obtained are summarized in Table I.

\section{Interpretation of PSt Spectrum}

Figure 2 is the PSt spectrum, which is different from those of the other polymers; only one peak appears. This peak can be assigned to phenyl carbon because of its relatively large intensity. On the other hand, peaks of methine and methylene carbon should appear in this region (see Table I), so all peaks seem to have coalesced into this large peak. The peak intensity is proportional to the corresponding number of atoms. For example, in the case of

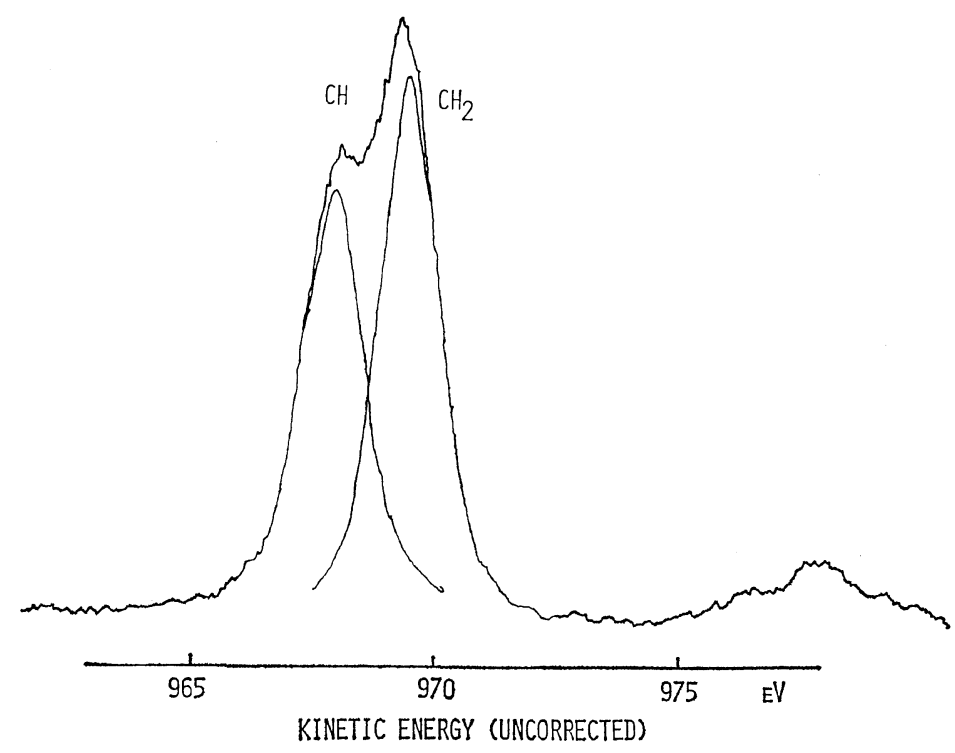

Figure 1. High-resolution ESCA spectrum of poly(vinyl alcohol) sample in CK region by $\mathrm{Mg}-\mathrm{K} \alpha$ radiation. Counting rate of the spectrum is about $10^{3} \mathrm{cps}$ at the maximum point. 
S. Suzuki, Y. Inoue, I. Ando, R. Chûjô, K. Seto, and K. Hoshino

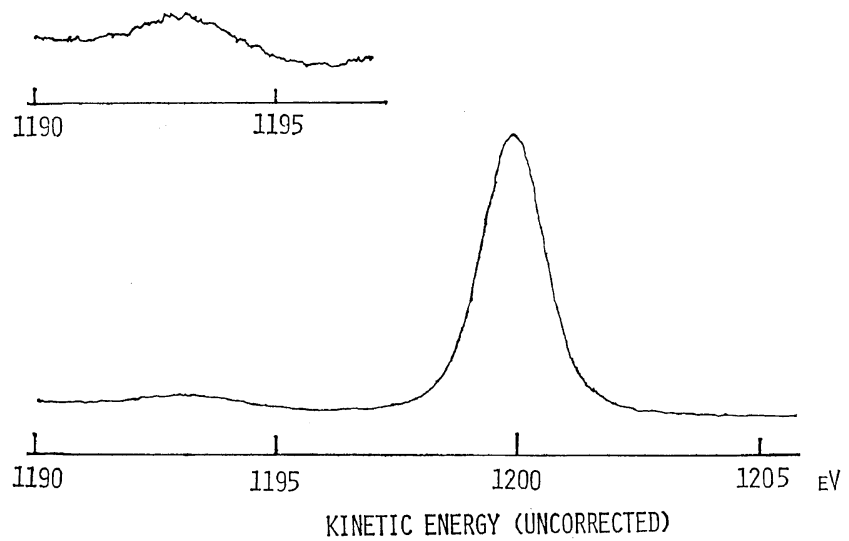

Figure 2. High-resolution ESCA spectrum of polystyrene sample in CK region by Al$\mathrm{K} \alpha$ radiation. Counting rate of the spectrum is about $5 \times 10^{3} \mathrm{cps}$ at the maximum point.

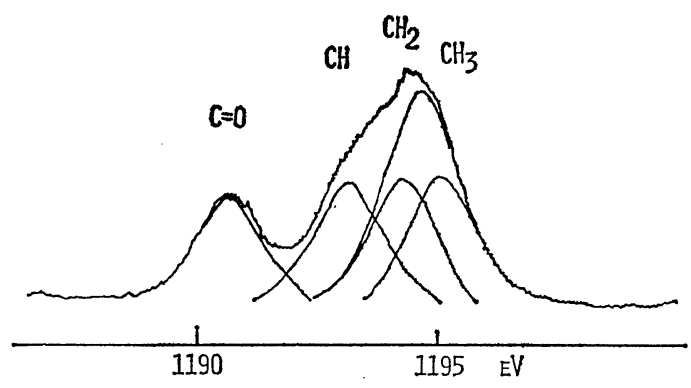

KINETIC ENERGY (UNCORRECTED)

Figure 3. High-resolution ESCA spectrum of poly(vinyl acetate) sample in $\mathrm{CK}$ region by $\mathrm{Al}-\mathrm{K} \alpha$ radiation. Counting rate of the spectrum is about $4 \times 10^{3} \mathrm{cps}$ at the maximum point.

PVAc, the relation of $1: 1: 1: 1$ is established between four peaks, i.e., methyl, methylene, methine, and carbonyl carbons, as shown in Figure 3. Another weak plateau appears in a position $7 \mathrm{eV}$ higher in binding energy than the main peak, as seen in Figure 2. A similar phenomenon is seen in the spectrum of $\operatorname{poly}(\alpha-$ methylstyrene), but the origin is as yet unknown.

\section{Influence of Contamination and Charging}

One of the disadvantages of ESCA spectra in the carbon and oxygen region is the possible coalescence of the spectra due to contamination of dust and oil in the instrument. In this experiment we cannot detect any such contamination influence. So even if there does exist some influence of contamination, its effect is not so
Table I. CK ESCA chemical shifts in linear polymers $^{\mathrm{a}}$

\begin{tabular}{|c|c|c|c|}
\hline No. & Polymer & $\begin{array}{l}\text { Chemical } \\
\text { species }\end{array}$ & $\begin{array}{l}\text { CK ESCA } \\
\text { chemical } \\
\text { shift, eV }\end{array}$ \\
\hline 1 & Polyacrylonitrile & Methylene & -0.6 \\
\hline 2 & & Methine & 0.3 \\
\hline 3 & & Cyano & 1.0 \\
\hline 4 & Poly(vinyl alcohol) & Methylene & -0.5 \\
\hline 5 & & Methine & 1.0 \\
\hline 6 & $\begin{array}{l}\text { Poly(methyl } \\
\text { methacrylate) }\end{array}$ & Carbonyl & 3.6 \\
\hline 7 & & Others & -0.4 \\
\hline 8 & Poly(vinyl chloride) & Methylene & -0.6 \\
\hline 9 & & Methine & 0.8 \\
\hline 10 & Polystyrene & Phenyl & -0.7 \\
\hline 11 & $\begin{array}{l}\text { Poly(ethylene } \\
\text { glycol) }\end{array}$ & Methylene & 1.0 \\
\hline 12 & Polyethylene & Methylene & 0.0 \\
\hline 13 & Poly(vinyl acetate) & Methyl & -0.8 \\
\hline 14 & & Methylene & 0.0 \\
\hline 15 & & Methine & 1.2 \\
\hline 16 & & Carbonyl & 3.7 \\
\hline 17 & Polypropylene & All & -0.4 \\
\hline
\end{tabular}

a The methylene carbon peak in polyethylene is adopted as the reference for chemical shifts. "Others" of PMMA and "All" of PP in the column of chemical species mean the average value of methylene, methyl, $\alpha$-skeltal, and methoxy carbons in the former, and methylene, methyl, and methine carbons in the latter.

large as to change the line shape of the spectra. Another disadvantage occurs when the samples 
are insulators like polymers, namely, a shift to the lower kinetic energy side due to charging. In order to correct this effect, gold was evaporated in vacuum on the sample surface. ${ }^{19} \mathrm{We}$ can safely say that this technique is useful for correcting the influence of charging. The values of binding energies in Table I are ones corrected by this technique.

Correlation between ESCA and NMR Chemical Shifts

Recently Swartz and Hercules ${ }^{1}$ investigated the correlation between ESCA and NMR chemical shifts of quaternary phosphonium compounds, and they indicated that a linear relation holds in general, with a few exceptions. Basch $^{20}$ and Zevoka ${ }^{21}$ theoretically derived a similar relation. The information obtained from ESCA and from NMR may be considered analogous in some sense, because ESCA measures the electrostatic shielding of a photoelectron, while NMR measures the magnetic shielding of a nucleus from an external field by electrons.

Figure 4 is a plot of CK ESCA chemical shifts against ${ }^{13} \mathrm{C}$ NMR chemical shifts. The latter values were adopted from previous reports. ${ }^{12-18}$ The straight lines (1) and (2) are obtained using a least square fit to all data in Table I except for point 10, and points 3, 6, 10 , and 16, respectively. If we can adopt the line (1) as a linear relation between ESCA and NMR chemical shifts, the datum from aromatic carbon (No, 10) does not fall on this line, while if the line (2) is adopted, the data from carbons with double- or triple-bonds $(3,6,10$, and 16)

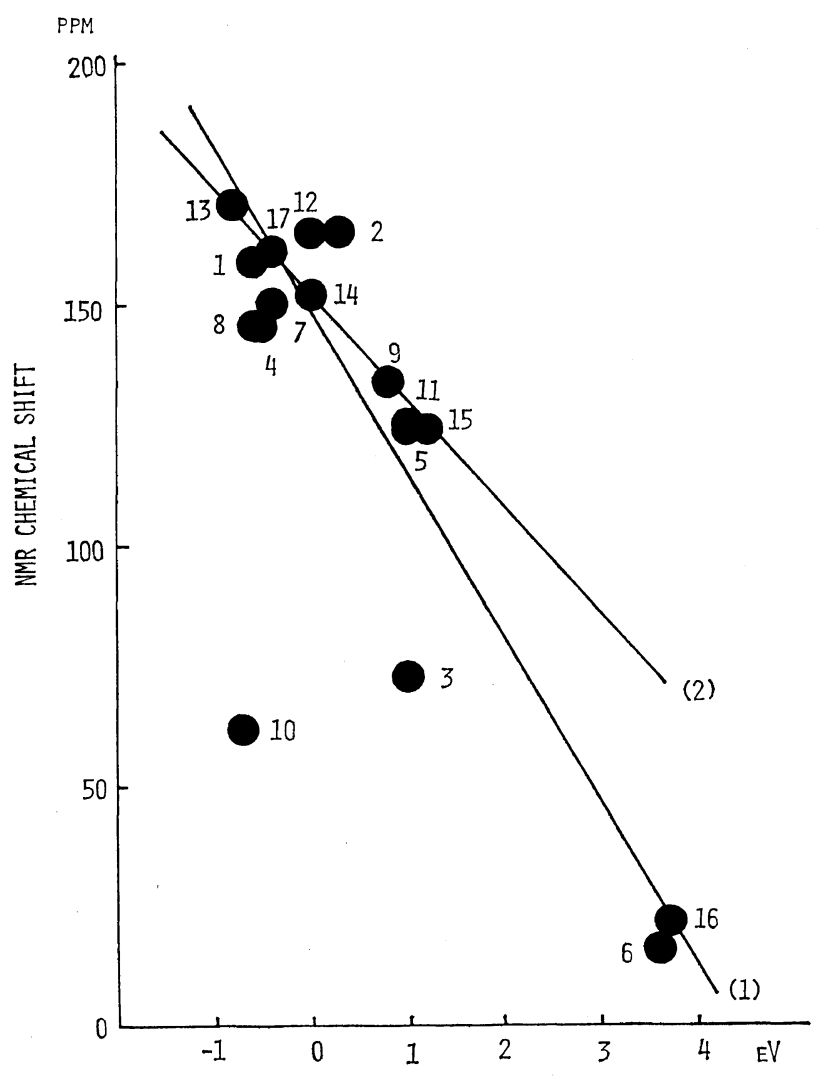

ESCA CHEMICAL SHIFT

Figure 4. Correlation between CK ESCA chemical shifts and ${ }^{13} \mathrm{C}$ NMR chemical shifts in linear polymers. The carbon peak of $\mathrm{CS}_{2}$ is adopted as the reference for NMR chemical shifts. Numbers in this figure are identical with those in Table I. 
do not fall on this line. This may be explained as follows. Owing to the delocalized $\pi$-electrons, the binding energies of carbons with unsaturated bonds become smaller than those expected for an aliphatic group. Which line is more appropriate is a question for further study. We can now safely say that a more or less linear relationship holds between CK ESCA chemical shifts and ${ }^{13} \mathrm{C}$ NMR chemical shifts of polymers. From Figure 4 we have the following equations between ESCA chemical shifts $\Delta E_{\mathrm{b}}$ and NMR chemical shifts $\delta_{\mathrm{c}}$ in the polymers examined:

$$
\begin{array}{ll}
\Delta E_{\mathrm{b}}=-0.029 \delta_{\mathrm{c}}+4.35 & \text { (from line (1)) } \\
\Delta E_{\mathrm{b}}=-0.045 \delta_{\mathrm{c}}+6.85 & \text { (from line (2)) }
\end{array}
$$

Correlation between ESCA Chemical Shifts and

\section{Electron Densities}

ESCA chemical shifts are usually explained by the electronegativities of the substituted groups. ${ }^{2,23}$ Generally in simple molecules this

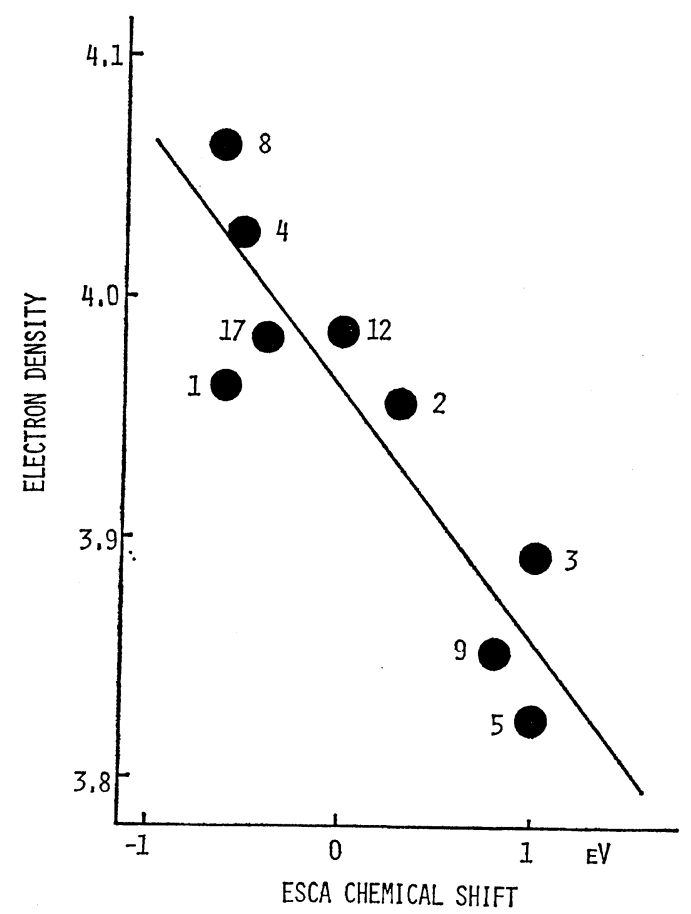

Figure 5. Correlation between CK ESCA chemical shifts and electron densities of the model compound. Electron densities are calculated by CNDO/2 method, except for PVC (extended Hückel method). Numbers in this figure are identical with those in Table I. explanation succeeds fairly well, but in more complex molecules agreement between experimental data and calculated points is not so good. For polymers no study of the correlation between ESCA chemical shifts and electron densities has been published. Figure 5 is a plot of CK ESCA chemical shifts against electron densities of model compounds (2,4-disubstituted pentane) calculated by $\mathrm{CNDO} / 2$ method, except for PVC (extended Hückel method). ${ }^{24}$ A straight line can be drawn using a least square method to all data in Figure 5. As a whole a linear relation holds. These calculated electron densities are only for $2 s$ and $2 p$ orbital electrons in carbon, while ESCA chemical shifts are for $\mathrm{CK}$ electrons. The slope of the straight line is in the sense which one would theoretically predict, since the larger electron density yields the lower binding energy, i.e., the smaller ESCA chemical shift. From this figure, we have the following equation between ESCA chemical

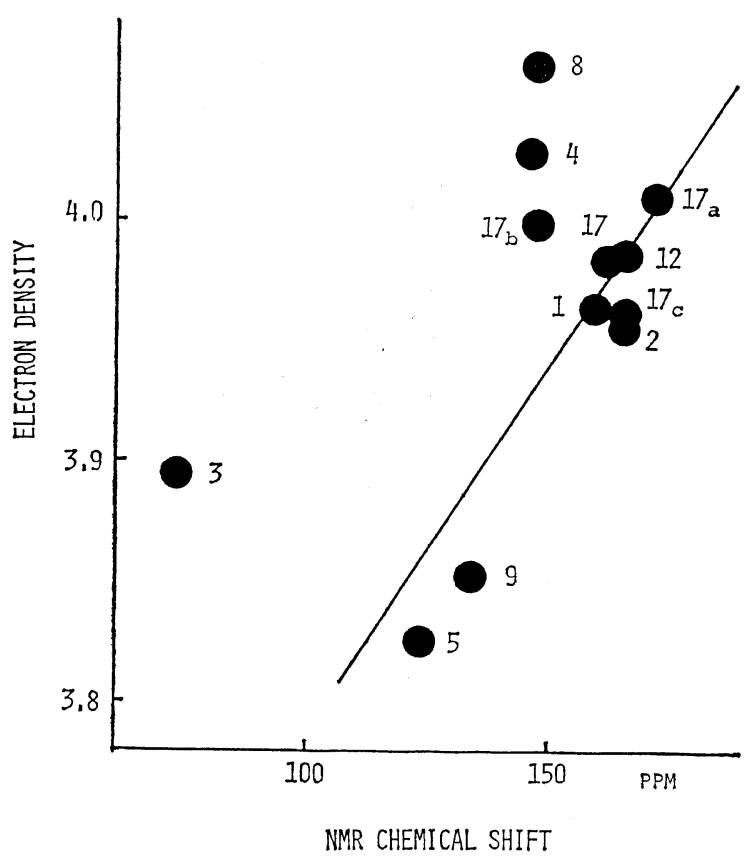

Figure 6. Correlation between ${ }^{13} \mathrm{C}$ NMR chemical shifts and electron densities of the model conpound. In this figure No. $17_{\mathrm{a}}, 17_{\mathrm{b}}$, and $17_{\mathrm{c}}$ mean methyl, methylene, and methine, carbon in polypropylene, and No. 17 is the average of these three values. Numbers in this figure are identical with those in Table I. 
shifts $\Delta E_{\mathrm{b}}$ and electron densities $E D$ :

$$
\Delta E_{\mathrm{b}}=-9.43 E D+37.38
$$

\section{Correlation between NMR Chemical Shifts and} Electron Densities

Figure 6 is a plot of ${ }^{13} \mathrm{C}$ NMR chemical shifts against electron densities. The straight line was drawn using a least square method to all data in Figure 6 except for points 3 and 8 . As a whole linear correlation is not seen; none the less, the larger electron densities yield the higher NMR chemical shifts, because the magnetic shielding of the atom increases. The equation of this line is

$$
\delta_{\mathrm{c}}=323 E D+1120
$$

Point No. 3 is particularly far away from the straight line and it thus contrasts with the agreement in Figure 5. So another factor may exist in NMR chemical shifts.

\section{CONCLUSIONS}

1. To correct the influence of charging of insulator-like polymers it is useful to evaporate metals like gold over the sample surface.

2. Two possible equations are found between ESCA chemical shifts $\Delta E_{\mathrm{b}}$ and NMR chemical shifts $\delta_{\mathrm{c}}$, as follows:

$$
\begin{array}{ll}
\Delta E_{\mathrm{b}}=-0.029 \delta_{\mathrm{c}}+4.35 & \text { (from line (1)) } \\
\Delta E_{\mathrm{b}}=-0.045 \delta_{\mathrm{c}}+6.85 & \text { (from line (2)) }
\end{array}
$$

It must be determined later which line is more appropriate.

3. One equation is found between ESCA chemical shifts $\Delta E_{\mathrm{b}}$ and electron densities $E D$, as follows:

$$
\Delta E_{\mathrm{b}}=-9.43 E D+37.38
$$

This relation holds good even for the carbons with unsaturated bonding studied in this report. The question whether all polymers obey the above relationship must await further experiments.

\section{REFERENCES}

1. W. E. Swartz, Jr., and D. H. Hercules, Anal.
Chem., 43, 1066 (1971).

2. R. E. Davis, D. L. Rousseau, and R. D. Board, Science, 171, 167 (1971).

3. R. E. Block, J. Magn. Resonance, 5, 155 (1971).

4. U. Gelius, G. Johansson, H. Siegbahn, C. J. Allan, D. A. Allison, J. Allison, and K. Siegbahn, J. Electron Spectrosc., 1, 285 (1972/ 73).

5. K. Maeda and K. Hoshino, The 24th Annual Meeting of the Chemical Society of Japan, 8212, 1971.

6. R. Chûjô, K. Sato, and A. Nishioka, Polymer J., 3, 242 (1972).

7. R. Chûjô, K. Maeda, K. Okuda, N. Murayama, and K. Hoshino, Rep. Prog. Polym. Phys. Japan, 15, 479 (1972).

8. M. Millard, International Conference of Electron Spectroscopy, Asilomar, Calif., 1971.

9. D. T. Clark and D. Kilcast, Nature. Phys. Sci., 233, 77 (1971).

10. D. T. Clark, W. J. Feast, D. Kilcast, and W. K. R. Musgrave, J. Polym. Sci. (Polym. Chem. Ed.), 11, 389 (1973).

11. D. T. Clark, D. Kilkast, W. J. Feast, and W. K. R. Musgrave, J. Polym. Sci. Part A-1, 10. 1637 (1972).

12. For polyacrylonitrile: Y. Inoue and A. Nishioka, Polymer J., 3, 149 (1972).

13. For poly(vinyl alcohol) and poly(vinyl acetate); Y. Inoue, R. Chûjô, A. Nishioka, S. Nozakura, and H. Iimura, ibid., 4, 244 (1973).

14. For poly(methyl methacrylate); Y. Inoue, A. Nishioka, and R. Chûjô, ibid., 2, 535 (1971).

15) For poly(vinyl chloride): Y. Inoue, I. Ando, and A. Nishioka, ibid., 3, 246 (1972).

16. For polystyrene: Y. Inoue, A. Nishioka, and R. Chûjô, Makromol. Chem., 156, 207 (1972).

17. For polyethylene: Y. Inoue, A. Nishioka, and R. Chûjô, ibid., 168, 163 (1973).

18. For polypropylene: Y. Inoue, A. Nishioka, R. Chûjô, ibid., 152, 15 (1972).

19. D. T. Hnatowich, J. Hedis, M. L. Perlman, and R. C. Ragaini, J. Appl. Phys., 42, 4883 (1971).

20. H. Basch, Chem. Phys. Lett., 5, 337 (1970).

21. D. Zeroka, ibid., 14, 471 (1972).

22. T. D. Thomas, J. Amer. Chem. Soc., 92, 4184 (1970).

23. R. Nordberg, H. Brecht, R. G. Albridge, A. Fahlman, and J. R. Wazar, Inorg. Chem., 9, 2469 (1970).

24. I. Ando, et al., unpublished. 